\title{
Prediction of hospital bed capacity during the COVID- 19 pandemic
}

\author{
Mieke Deschepper ${ }^{1 *} \mathbb{D}$, Kristof Eeckloo ${ }^{1,2}$, Simon Malfait ${ }^{1}$, Dominique Benoit ${ }^{3}$, Steven Callens ${ }^{4}$ and \\ Stijn Vansteelandt 5,6
}

\begin{abstract}
Background: Prediction of the necessary capacity of beds by ward type (e.g. ICU) is essential for planning purposes during epidemics, such as the COVID- 19 pandemic. The COVID- 19 taskforce within the Ghent University hospital made use of ten-day forecasts on the required number of beds for COVID- 19 patients across different wards.

Methods: The planning tool combined a Poisson model for the number of newly admitted patients on each day with a multistate model for the transitions of admitted patients to the different wards, discharge or death. These models were used to simulate the required capacity of beds by ward type over the next 10 days, along with worstcase and best-case bounds.

Results: Overall, the models resulted in good predictions of the required number of beds across different hospital wards. Short-term predictions were especially accurate as these are less sensitive to sudden changes in number of beds on a given ward (e.g. due to referrals). Code snippets and details on the set-up are provided to guide the reader to apply the planning tool on one's own hospital data.
\end{abstract}

Conclusions: We were able to achieve a fast setup of a planning tool useful within the COVID- 19 pandemic, with a fair prediction on the needed capacity by ward type. This methodology can also be applied for other epidemics.

Keywords: COVID-19, Multistate modeling, Poisson modelling, Hospital data

\section{Background}

The enormous impact of the COVID-19 pandemic has surprised many hospitals beginning March 2020. It soon became apparent that the capacity of hospital beds was at the verge of coming under great pressure. Besides a shift of regular beds to specific COVID-19 beds with special hygiene measures, pressure on the number of beds arose primarily from the need to foresee sufficient capacity in the Intensive Care Unit (ICU). Indeed, while approximately 9 to $11 \%$ of admitted COVID-19 patients were in need of advanced life-supporting measures [1], ICU capacity was limited in terms of the number of

\footnotetext{
* Correspondence: Mieke.deschepper@uzgent.be

${ }^{1}$ Strategic Policy Cell at Ghent University Hospital, C. Heymanslaan 10, 9000 Ghent, Belgium

Full list of author information is available at the end of the article
}

beds, but also the number of monitoring devices, life supporting machines and specific trained personnel to provide high quality of care. In the Belgian situation, which we will consider, the number of ICU beds is on average 15.9 per 100.000 inhabitants; it is less favourable in the rest of Europe, numbering 11.5 per 100.000 inhabitants [2]. For healthcare systems, and hospitals within these systems, organizational preparedness and capacity planning was thus essential [3]. In Belgium, for instance, a Surge Capacity Plan [4] has been set up to monitor the number of occupied ICU beds and to create extra ICU beds where needed. In cases of (near) saturation, contact had to be made with the local health inspector. Moreover, so long as saturation was threatened at the national level, a certain percentage of the beds needed to be allocated and foreseen for potential COVID-19 patients

(C) The Author(s). 2021 Open Access This article is licensed under a Creative Commons Attribution 4.0 International License, which permits use, sharing, adaptation, distribution and reproduction in any medium or format, as long as you give appropriate credit to the original author(s) and the source, provide a link to the Creative Commons licence, and indicate if changes were made. The images or other third party material in this article are included in the article's Creative Commons licence, unless indicated otherwise in a credit line to the material. If material is not included in the article's Creative Commons licence and your intended use is not permitted by statutory regulation or exceeds the permitted use, you will need to obtain permission directly from the copyright holder. To view a copy of this licence, visit http://creativecommons.org/licenses/by/4.0/ The Creative Commons Public Domain Dedication waiver (http://creativecommons.org/publicdomain/zero/1.0/) applies to the data made available in this article, unless otherwise stated in a credit line to the data. 
(described in different phases by the government). To attain the required capacity, patients in burn units were centralized at the national level.

Hospital capacity planning is driven by complex dynamics between input, output and the number of available beds [5, 6]. In normal times, hospitals aim to achieve an optimal bed occupancy by maximizing bed occupancy while minimizing overflow, which often has a negative effect on patient outcomes [7]. However, pandemics and natural disasters typically come with a sudden influx of unforeseen patients, which almost instantly pushes the boundaries of a hospital's capacity [8]. Frontline health care workers, directly engaged in the diagnosis, treatment, and care for patients with COVID-19, are susceptible to experience psychological burden in return, while also being at greater health risks [9]. Lack of bed capacity, scarcity in supplies and high occupancy rates further increases that burden.

In order to prevent such overflow, healthcare systems can take several measures. In China, new hospitals have been built [10], which immediately increased capacity via a larger number of available beds. However, most European countries underestimated the pandemic potential and virulence, and as such did not take such actions. In most countries, the influx in hospitals was instead reduced by means of a nationwide quarantine, measures of social distancing, hand washing, school closures, mouth mask or other activities [3]. Such measures successfully flattened the curve, decreasing the influx and therefore putting less stress on hospital capacity.

However, successfully flattening the curve means extending the duration of the pandemic, making it impossible to further postpone regular care [11]. A fragile equilibrium needs to be found between reserving a sufficient number of beds for COVID-19 cases, while also providing sufficient beds for regular, necessary care which cannot be delayed. In order to achieve such balance, predictive models can play an important role, not only to predict the number of needed beds that should be allocated to the pandemic, but also to inform the hospital on providing the right equipment and training sufficient healthcare workers for specific cases [12].

The ability to predict hospital bed capacity for different types of wards is essential for monitoring and planning purposes during epidemics, such as the ongoing COVID-19 pandemic. At the start of the pandemic, the available models were scarce. Most efforts then focused on susceptible-infected-removed (SIR) models, and variations thereof, aimed at predicting the number of positive COVID-19 cases at a national level (e.g. [13, 14]). While these provide valuable insights into the dynamics of future disease spreading in a population, we could not readily make use of those for hospital planning because they demand input on parameters, such as doubling times and social distancing measures, that were not available for our local setting at that time. Moreover, they are not designed to give detailed predictions for a specific hospital, organized by type of ward. A further limitation is that standard SIR models not accounting for cohort structure underestimate the peak of COVID19 infectious cases and their timing [15]. General purpose simulation toolboxes, such as the (free) web application corona.simbox.ai, predict capacity building on observed trends in the number of new cases and the expected length of hospital stay observed in specific countries (data from https://www.worldometers.info/ coronavirus/). While potentially more directly useful, their generic nature has the disadvantage of providing capacity predictions that are not well aligned with the regional variation in the severity of the epidemic, local treatment, triage and hospital management policies. Within the Ghent University Hospital, we have therefore set up a planning tool to predict on each day the needed capacity of different bed types over the subsequent tenday period. Based on the tool's predictions, the required human capacity (i.e. healthcare workers) can be assessed and the needed material can be stocked. Such capacity planning forms an essential primordial step in preparing a hospital. In particular, we will develop a data-driven prediction algorithm which makes use of daily updated hospital records to make predictions on each day, of the number of new cases that can be expected over the next 10 subsequent days, as well as how admitted cases are expected to transition during this period between different wards, as well as to discharge or death. The proposed algorithm makes use of Poisson models with smoothing splines to model the evolution in the number of new cases over time, along with multistate models [16] to describe patient transitions between multiple states (namely, wards, discharge or death). These fitted models, which are daily updated, are then used to simulate the capacity needed over the subsequent 10 days.

\section{Methods \\ Population}

The data includes all patients admitted to the University Hospital Ghent and labelled as COVID-19 patient; some of these are transferals from other hospitals. We label a patient as a COVID-19 patient, when a positive PCR test in the lab (internal or external) is present. Some patients in our dataset had already been admitted before obtaining a positive PCR test result (e.g. in the Rehabilitation department). We use data from different time points from one hospital and use as such a longitudinal study design.

\section{Inclusion criteria}

The model is trained on all positive COVID-19 patients who were in the University Hospital Ghent before April 
20,2020 . We test the model on the patients who were in the hospital between April 20, 2020 and April 27, 2020. This range of dates falls within the peak of the pandemic and refers to one of the first weeks the model was fully operational.

\section{Statistical modelling}

The implementation of the different steps can be found in the Supplementary Appendix, along with code snippets.

\section{Multistate model}

We use multistate modelling to model the time for current patients needed to transition to a different ward, as well as to discharge or hospital death. In particular, we model transitions between the wards Non-Covid19, Cohort, ICU Midcare, ICU Standard and ICU Ventilated. Here, Non-Covid19 includes all the wards without positive COVID-19 patients, such as the Emergency Department, but also the wards where non-COVID-19 patients stay during the pandemic. Cohort includes all wards with COVID-19 patients who need standard care. The task force decided to open a specific COVID-19 midcare unit (ICU Midcare), to better guarantee availability of Intensive Care department (ICU) beds for the most severely ill patients with a good chance of ICU survival. The ICU was further divided into unventilated critically ill patients (ICU Standard) and ventilated critically ill patients (ICU Ventilated). We chose to split these two types of ICU wards to enable capacity planning on the required number of ventilators. Our model makes no distinction between discharge or death, as it has no consequences for capacity planning.

Multistate models describe events over the course of time as transitions between multiple states. A first step is to define all possible transitions (Fig. 1, Appendix A1). Patients arrive at Non-Covid19 (e.g. emergency department), from which they can be transferred to Cohort (= all non-ICU wards with COVID-19 patients), ICU Midcare, ICU Standard or ICU Ventilated. Each patient can have multiple transfers between the different wards, terminating in state Discharged, which indicates that the patient either has been discharged or has died.

The considered multistate model places no constraints on the possible transitions that can be made. In particular, the cause-specific hazard of each transition is modelled non-parametrically and estimated using the AalenJohansen estimator [17]. This is done under a standard Markovian assumption that the hazard to transition to a given state (e.g. ward), while possibly different depending on the current state in which the patient is present, has no residual dependence on earlier states in which the patient was observed. For instance, the cause-specific hazard to transition to Cohort after having spent 10 days on ventilation is assumed to be the same, regardless of whether the patient was already in the ICU prior to ventilation, versus was directly admitted to ICU Ventilated. To enable a fast implementation and because covariate data of future cases are obviously missing, no covariate adjustment is made in these models.

All models were fitted in $\mathrm{R}$ (version 3.6.1), using the implementation from the mstate [18] package for multistate models (see Appendix A2).

\section{Simulating transition for patients already present in the hospital}

The fitted multistate model was used to simulate on each day of the pandemic, how COVID-19 patients currently present in the hospital are expected to transition to other wards, discharge or death. In particular, for each patient, we calculated their cause-specific hazard to transition to each of the other wards, discharge or death on each of the subsequent 10 days, based on their latest state and the time already spent in that state. Based on these estimated cause-specific hazards, the patient's possible transitions through the different states were then randomly simulated. Subsequently, the number of occupied beds on each ward was calculated for each of the subsequent 10 days. This process was repeated $M$ times (in our case 500) in order to eliminate simulation error

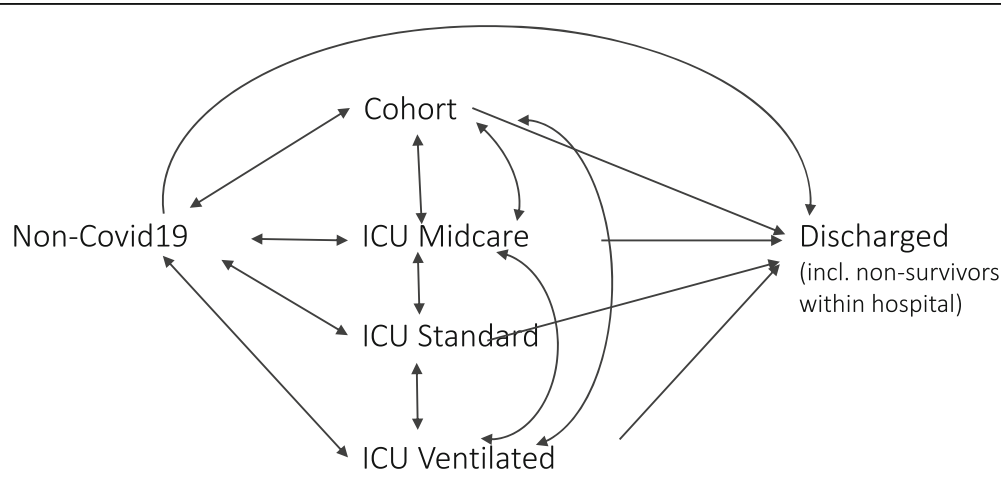

Fig. 1 Possible transitions in the multistate model 
as well as to develop insight into the degree of uncertainty. Simulations were based on the $\mathrm{R}$ function mstate::mssample (see Appendix A2.4).

\section{Simulating transitions for new patients}

To predict the number of new cases expected over the subsequent 10 day(s), we use additive Poisson modelling. In particular, we model the logarithm of the number of daily new cases using a Poisson model with a penalized regression spline for calendar time. Smoothing parameters are selected based on Mallow's Cp. All models are fitted in $\mathrm{R}$ (version 3.6.1), using the implementation from the $m g c v$ [19] package for additive Poisson modelling (Appendix A4).

Based on the fitted Poisson model, we next simulate the number of new cases that is expected to arrive on each of the coming 10 days. For convenience, these patients are assumed to enter the Non-Covid19 ward (such as ER) ( $\mathrm{R}$ package $m g c v:: g a m$ ), with their time set to zero. Next, the fitted multistate model is used to simulate how new cases will transition to different wards, discharge or death over the coming 10 days. Also this entire simulation process was repeated five hundred times. In doing so, we accounted for the fact that e.g. for a patient who was simulated to be newly admitted on day eight, we only need to simulate his/her transitions for the subsequent two days (see Table 1).

The total number of occupied beds across existing and new patients was calculated for each of the subsequent
10 days in each of the $M$ simulation runs. The obtained results were averaged across the $M$ simulation runs to eliminate simulation error. In addition, to summarize the uncertainty in the possible capacity needed on each day, we report a best case scenario (corresponding to the $5 \%$ percentile of the needed capacity) and a worst scenario (corresponding to the $95 \%$ percentile of the needed capacity).

To assess the degree of inaccuracy in the results stemming from the limited number of simulation runs, we calculated Monte Carlo simulation error. For the mean scenario, this is given by the standard error of the mean (defined as the standard deviation of the capacity across the $\mathrm{M}$ simulations, divided by the square root of the number of simulations). For the two percentiles, we report the standard error calculated using Nyblom's interpolated order statistic approach [20] (available from the R package quantileCI::quantile_confint_nyblom) (Appendix A3).

\section{Model validation}

To validate the model we compare the bed occupancy predicted on April 20, 2020 and April 27, 2020, which corresponds with the first peak of the pandemic, with the actual values. We also evaluate one-day-ahead predictions in the period in between these two dates. This means that for every day a new prediction is made for just the next day, e.g. on April 23, 2020 the prediction is made for April 24, 2020 and on April 24, 2020 the prediction is made for April 25, 2020.

Table 1 Example table of the predictions made for each day the next coming ten days

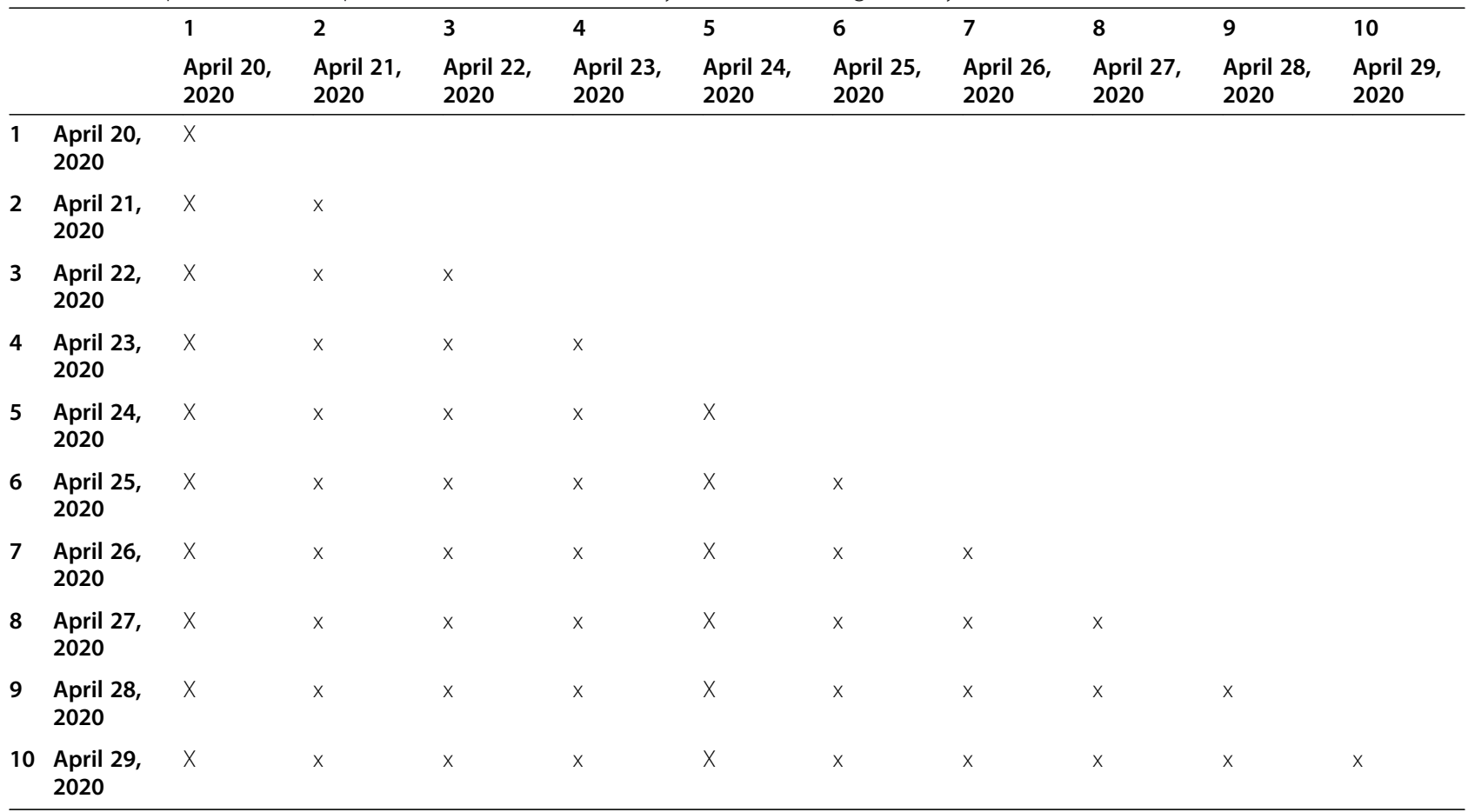

Legend: An $\mathrm{x}$ represents 500 simulations in our analysis 
Table 2 Patient characteristics for April 20, 2020 and April 27, 2020

\begin{tabular}{lll}
\hline & April 20, 2020 & April 27, 2020 \\
\hline$N$ (number included admissions) & 203 & 222 \\
Gender (male) & $130(64 \%)$ & $139(63 \%)$ \\
Age $[S D]$ & $56.84[17.51]$ & $57.07[17.72]$ \\
Patient status at moment of data collection & \\
$\quad$ Non-survivors & $21(10 \%)$ & $24(11 \%)$ \\
$\quad$ Discharged & $123(61 \%)$ & $145(65 \%)$ \\
$\quad$ Non-discharged & $59(29 \%)$ & $53(24 \%)$ \\
\hline
\end{tabular}

Legend: SD Standard Deviation

\section{Results}

\section{Patient characteristics}

We use and apply this approach on data from the Ghent University hospital during the COVID-19 pandemic. On April 20, 2020 this dataset consists of 203 different people in hospital, while on April 27, 2020222 admissions are included. More males than females are admitted with an average age of sixty (Table 2). At the two selected time points, a large fraction of all patients (29$24 \%)$ is still in hospital.

\section{Multistate models}

We estimate the overall transition probabilities (using the mstate::probtrans function) from the multistate model (fit with mstate::msfit) (Appendix A1). In Fig. 2 we can see the overall transition probabilities for the ward in which we wish to predict the number of patients by day, which express what percentage of patients is expected in each state in function of the number of days since admission. It shows a majority of patients in
Cohort, and moreover indicates long length of stay on ICU Ventilated.

\section{Simulating transition for all patients}

The results of the Poisson modelling to predict the number of expected patients for the next ten days is given in Fig. 3. We show the actual numbers in dark bars and the predicted number of new patients in lighter boxplots. For interpretation of the boxplots we refer to Appendix A5.

The results of the simulations are visualized in bar charts and in a table with the absolute numbers of patients. The bar chart (Fig. 4) shows the trends as well as the difference between actual and predicted number of patients; the table (Table 3 ) holds the absolute numbers which are of interest to stakeholders. The graph and table also display simulation error, which is small, indicating that 500 simulation runs suffice. As expected from the transition probabilities, the largest proportion of patients is expected to stay in Cohort. This number first increases, as this is where new patients arrive before possible transfer to other wards. We observe a similar effect on ICU Midcare, where transfers from Cohort and ICU Ventilated result in an increase of patients on this ward and transfers back or to these same wards for a decrease. The evolution on ICU Ventilated is different, with patients tending to have long lengths of stay once admitted. ICU Standard has a very limited number of patients.

\section{Model validation}

To validate our model, we compare the actual and predicted numbers of patients (Table 4).

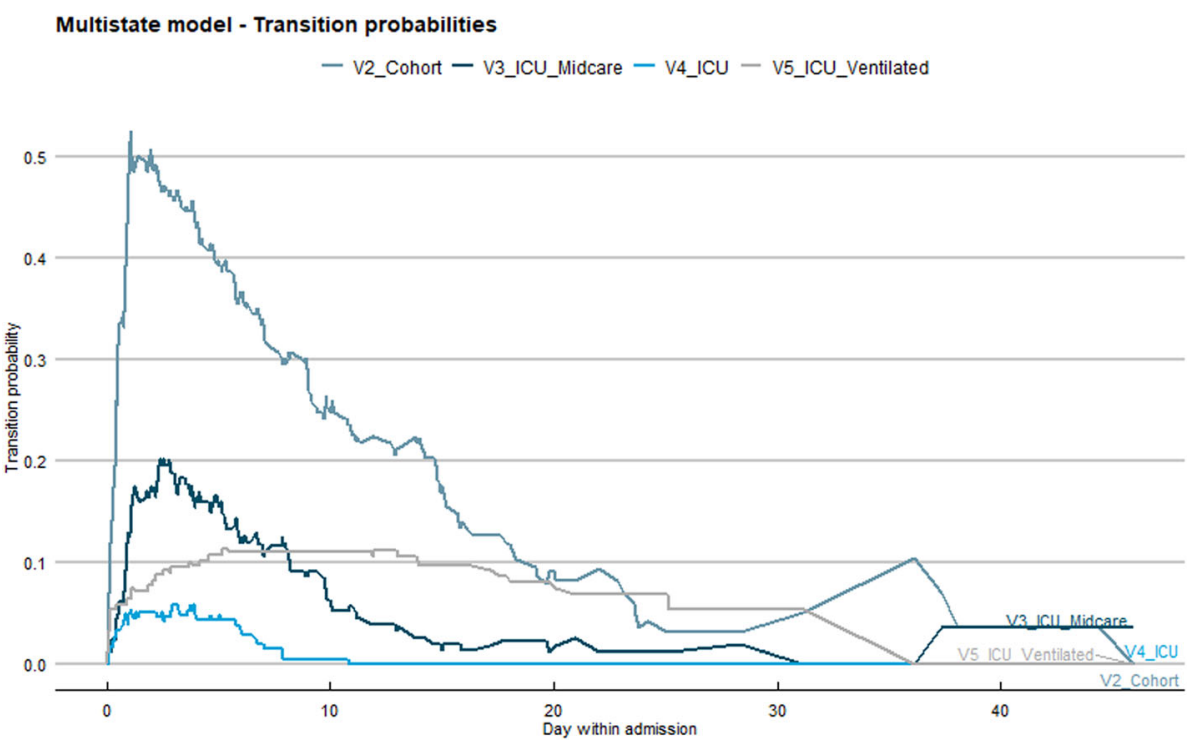

Fig. 2 Transition probabilities: probabilities to transition from the first state (Non-Covid19) to selected states over the considered time window 
Number of admission by day

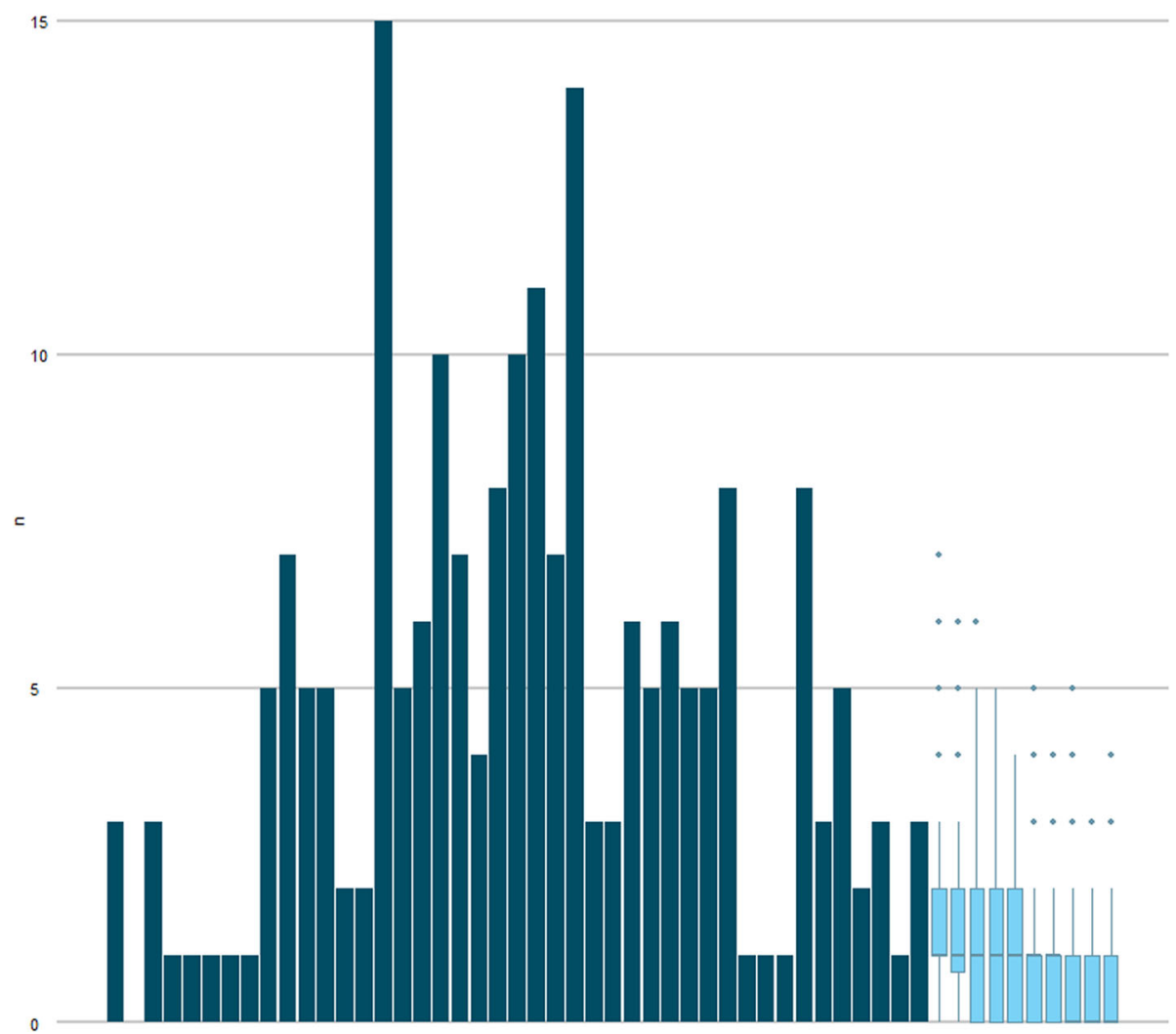

Fig. 3 Observed and predicted number of new patients - Poisson modelling - April 20, 2020. The dark bars are the actual numbers of new admission by day. The lighter boxplots show the predicted numbers of expected admissions by day

Where Table 4 shows the prediction for the next 10 days, Table 5 shows the prediction for just the next day. If the actual difference on one day is large, this has a negative effect on the prediction (e.g. April 22, 2020 ICU Midcare). This has no effect on the prediction for the days after, as the actual numbers are included in the dataset. Nevertheless, this does imply that for prediction on multiple days (e.g. 10 days as in Table 4) will results in an unexpected change that will be lower or higher than expected.

The large difference on April 28, 2020 in ICU Ventilated is caused by a sudden two deaths and three transfers.

\section{Discussion}

In this paper, we have proposed an algorithm that can be used for capacity planning during an epidemic, along with software code. We are not aware of similar data driven approaches that fully rely on one's own hospital health records.

We have found the proposed approach to be fairly reliable in predicting the required capacity within Ghent
University hospital, except at the start of the pandemic where the number of data is still too limited to enable reliable prediction, and where the organization may not be in a sufficiently stable situation to enable extrapolation to the future. For this, it can be useful to borrow strength by combining data across multiple regional hospitals. Also, it can be used in a second wave, using the parameters from the first wave. This would then become more specific for the institution, as physicians and treatment protocols differ among the different institutions.

While using this tool during the COVID-19 pandemic, we have found the number of patients on ICU Midcare to be the hardest to predict (Table 5), where we observe between -3 and +5 deviation between the actual and predicted numbers. The reason is that this ward receives input and output from Cohort as from ICU Standard/ ICU Ventilated, making the numbers of patients on this ward very sensitive to human decisions made on these other wards, and possibly even the insight of a single physician. Our results, including the ones reported in this paper (and used by the task force of the Ghent University hospital), are based on predictions made on 


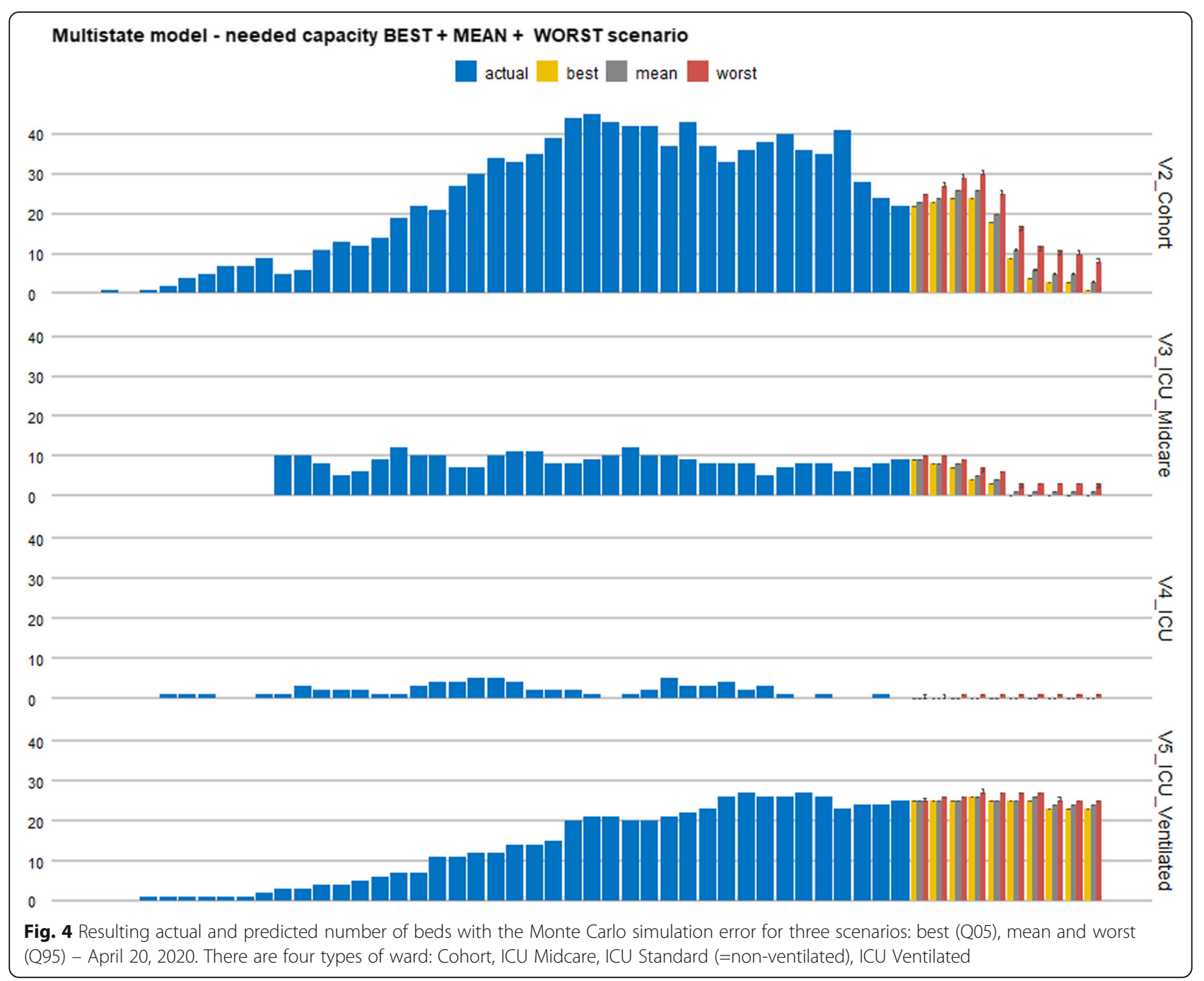

Mondays. These are subject to a possible weekend effect, as decisions on opening/closing/changing wards were usually made just prior to the weekend and this could influence the results. These decisions mainly related to shifting or changing ICU Midcare, adding to the difficulty of predicting the capacity on ICU Midcare on Mondays.

A further limitation of our proposal is that the Poisson model may need some time to pick up sudden increases or drops in the expected number of new cases, e.g. due to a relaxation of lockdown regulations. The use of smoothing splines allows sufficient flexibility to pick up such effects, but some time is needed for this to be picked up in a reliable way.

The proposed approach is simulation-based, which is useful to develop insight into random fluctuations that may occur in the required capacity. The calculated Monte Carlo simulation error suggested 500 simulations to suffice in order to dampen simulation error. Model validation was based on an independent dataset as the predictions were evaluated on future data not known upfront or used within the training set data.

Our reported best- and worst-case scenario ignore the excess variability that may arise from the fact that the Poisson and multistate models were themselves fitted on limited data and are thus subject to imprecision, making the reported intervals somewhat optimistic. Acknowledging this excess variability is non-trivial, and beyond the scope of this work.

The proposed approach is transposable to any other epidemic or very specific pathology/disease for which one is interested to know the required number of beds for specific wards and with a specific flow. As most administrative systems will have at least one row for every transfer for all patients, these can be bundled and as such used in the same approach as described. The extent of data manipulation should not be underestimated as each system has its own layout / structured and is difficult to generalize internationally (in Belgium most 
Table 3 Result table

\begin{tabular}{|c|c|c|c|c|c|c|c|c|c|c|c|c|}
\hline \multirow[t]{2}{*}{ day } & \multicolumn{3}{|l|}{ Cohort } & \multicolumn{3}{|c|}{ ICU Midcare } & \multicolumn{3}{|c|}{ ICU Standard } & \multicolumn{3}{|c|}{ ICU Ventilated } \\
\hline & & Actual & & & Actual & & & Actual & & & Actual & \\
\hline 2020-04-15 & & 36 & & & 8 & & & 0 & & & 27 & \\
\hline 2020-04-16 & & 35 & & & 8 & & & 1 & & & 26 & \\
\hline 2020-04-17 & & 41 & & & 6 & & & 0 & & & 23 & \\
\hline 2020-04-18 & & 28 & & & 7 & & & 0 & & & 24 & \\
\hline \multirow[t]{2}{*}{ 2020-04-19 } & & 24 & & & 8 & & & 1 & & & 24 & \\
\hline & Best & Median & Worst & Best & Median & Worst & Best & Median & Worst & Best & Median & Worst \\
\hline $\begin{array}{l}2020-04- \\
20\end{array}$ & $\begin{array}{c}24 \\
{[24-24]}\end{array}$ & $\begin{array}{c}25 \\
{[24.96-} \\
25.04]\end{array}$ & $\begin{array}{c}27 \\
{[27-28]}\end{array}$ & $\begin{array}{c}10 \\
{[10-} \\
10]\end{array}$ & $\begin{array}{c}10 \\
{[9.98-} \\
10.02]\end{array}$ & $\begin{array}{c}11 \\
{[10.6-11]}\end{array}$ & $\begin{array}{c}1 \\
{[1-} \\
1]\end{array}$ & $\begin{array}{c}1 \\
{[0.98-} \\
1.02]\end{array}$ & $\begin{array}{c}1 \\
{[1-1]}\end{array}$ & $\begin{array}{c}24 \\
{[24-} \\
24]\end{array}$ & $\begin{array}{c}24 \\
{[23.98-} \\
24.02]\end{array}$ & $\begin{array}{c}24 \\
{[24-26.13]}\end{array}$ \\
\hline 2020-04-21 & $\begin{array}{c}25 \\
{[25-26]}\end{array}$ & $\begin{array}{c}27 \\
{[26.91-} \\
27.09]\end{array}$ & $\begin{array}{c}30 \\
{[29-32]}\end{array}$ & $\begin{array}{c}8 \\
{[8-8]}\end{array}$ & $\begin{array}{c}9 \\
{[8.96-9.04]}\end{array}$ & $\begin{array}{c}10 \\
{[10-} \\
10.53]\end{array}$ & $\begin{array}{c}1 \\
{[1-} \\
1]\end{array}$ & $\begin{array}{c}1 \\
{[0.96-} \\
1.04]\end{array}$ & $\begin{array}{c}1 \\
{[1-} \\
3.32]\end{array}$ & $\begin{array}{c}24 \\
{[24-} \\
24]\end{array}$ & $\begin{array}{c}24 \\
{[23.96-} \\
24.04]\end{array}$ & $\begin{array}{c}25 \\
{[24.47-} \\
25.67]\end{array}$ \\
\hline $2020-04-22$ & $\begin{array}{c}27 \\
{[26.89-28]}\end{array}$ & $\begin{array}{c}30 \\
{[29.84-} \\
30.16]\end{array}$ & $\begin{array}{c}33 \\
{[32-} \\
33.11]\end{array}$ & $\begin{array}{c}6 \\
{[6-6]}\end{array}$ & $\begin{array}{c}7 \\
{[6.93-7.07]}\end{array}$ & $\begin{array}{c}8 \\
{[8-9]}\end{array}$ & $\begin{array}{c}1 \\
{[1-} \\
1]\end{array}$ & $\begin{array}{c}1 \\
{[0.93-} \\
1.07]\end{array}$ & $\begin{array}{c}1 \\
{[1-2]}\end{array}$ & $\begin{array}{c}25 \\
{[25-} \\
25]\end{array}$ & $\begin{array}{c}25 \\
{[24.93-} \\
25.07]\end{array}$ & $\begin{array}{c}26 \\
{[26-27]}\end{array}$ \\
\hline $2020-04-23$ & $\begin{array}{c}31 \\
{[31-32]}\end{array}$ & $\begin{array}{c}34 \\
{[33.8-34.2]}\end{array}$ & $\begin{array}{c}37 \\
{[37-} \\
39.33]\end{array}$ & $\begin{array}{c}5 \\
{[5-5]}\end{array}$ & $\begin{array}{c}6 \\
{[5.91-6.09]}\end{array}$ & $\begin{array}{c}8 \\
{[8-9]}\end{array}$ & $\begin{array}{c}1 \\
{[1-} \\
1]\end{array}$ & $\begin{array}{c}1 \\
{[0.91-} \\
1.09]\end{array}$ & $\begin{array}{c}1 \\
{[1-2]}\end{array}$ & $\begin{array}{c}26 \\
{[26-} \\
26]\end{array}$ & $\begin{array}{c}26 \\
{[25.91-} \\
26.09]\end{array}$ & $\begin{array}{c}27 \\
{[27-28]}\end{array}$ \\
\hline $2020-04-24$ & $\begin{array}{c}27 \\
{[26-27]}\end{array}$ & $\begin{array}{c}30 \\
{[29.76-} \\
30.24]\end{array}$ & $\begin{array}{c}34 \\
{[33-} \\
35.22]\end{array}$ & $\begin{array}{c}4 \\
{[4-4]}\end{array}$ & $\begin{array}{c}5 \\
{[4.89-5.11]}\end{array}$ & $\begin{array}{c}7 \\
{[7-8]}\end{array}$ & $\begin{array}{c}1 \\
{[1-} \\
1]\end{array}$ & $\begin{array}{c}1 \\
{[0.89-} \\
1.11]\end{array}$ & $\begin{array}{c}2 \\
{[1-2]}\end{array}$ & $\begin{array}{c}26 \\
{[26-} \\
26]\end{array}$ & $\begin{array}{c}26 \\
{[25.89-} \\
26.11]\end{array}$ & $\begin{array}{c}28 \\
{[28-28]}\end{array}$ \\
\hline $2020-04-25$ & $\begin{array}{c}23 \\
{[21.89-} \\
23.1]\end{array}$ & $\begin{array}{c}26 \\
{[25.72-} \\
26.28]\end{array}$ & $\begin{array}{c}30 \\
{[29-} \\
31.22]\end{array}$ & $\begin{array}{c}2 \\
{[2-2]}\end{array}$ & $\begin{array}{c}3 \\
{[2.88-3.12]}\end{array}$ & $\begin{array}{c}6 \\
{[5-6]}\end{array}$ & $\begin{array}{c}1 \\
{[1-} \\
1]\end{array}$ & $\begin{array}{c}1 \\
{[0.88-} \\
1.12]\end{array}$ & $\begin{array}{c}2 \\
{[2-2]}\end{array}$ & $\begin{array}{c}25 \\
{[25-} \\
25]\end{array}$ & $\begin{array}{c}26 \\
{[25.88-} \\
26.12]\end{array}$ & $\begin{array}{c}27 \\
{[27-27.18]}\end{array}$ \\
\hline $2020-04-26$ & $\begin{array}{c}15 \\
{[14-16]}\end{array}$ & $\begin{array}{c}19 \\
{[18.7-19.3]}\end{array}$ & $\begin{array}{c}23 \\
{[22-25]}\end{array}$ & $\begin{array}{c}1 \\
{[1-1]}\end{array}$ & $\begin{array}{c}3 \\
{[2.86-3.14]}\end{array}$ & $\begin{array}{c}5 \\
{[5-6]}\end{array}$ & $\begin{array}{c}1 \\
{[1-} \\
1]\end{array}$ & $\begin{array}{c}1 \\
{[0.86-} \\
1.14]\end{array}$ & $\begin{array}{c}2 \\
{[1-2]}\end{array}$ & $\begin{array}{c}25 \\
{[25-} \\
25]\end{array}$ & $\begin{array}{c}26 \\
{[25.86-} \\
26.14]\end{array}$ & $\begin{array}{c}27 \\
{[27-28]}\end{array}$ \\
\hline $2020-04-27$ & $\begin{array}{c}9 \\
{[8-9]}\end{array}$ & $\begin{array}{c}12 \\
{[11.7-12.3]}\end{array}$ & $\begin{array}{c}16 \\
{[15.9-18]}\end{array}$ & $\begin{array}{c}1 \\
{[1-1]}\end{array}$ & $\begin{array}{c}3 \\
{[2.87-3.13]}\end{array}$ & $\begin{array}{c}5 \\
{[5-5]}\end{array}$ & $\begin{array}{c}1 \\
{[1-} \\
1]\end{array}$ & $\begin{array}{c}1 \\
{[0.87-} \\
1.13]\end{array}$ & $\begin{array}{c}2 \\
{[1.19-} \\
2]\end{array}$ & $\begin{array}{c}25 \\
{[25-} \\
25]\end{array}$ & $\begin{array}{c}26 \\
{[25.87-} \\
26.13]\end{array}$ & $\begin{array}{c}28 \\
{[27-28]}\end{array}$ \\
\hline $2020-04-28$ & $\begin{array}{c}11 \\
{[9-11]}\end{array}$ & $\begin{array}{c}14 \\
{[13.7-14.3]}\end{array}$ & $\begin{array}{c}18 \\
{[17-20]}\end{array}$ & $\begin{array}{c}1 \\
{[1-1]}\end{array}$ & $\begin{array}{c}3 \\
{[2.87-3.13]}\end{array}$ & $\begin{array}{c}5 \\
{[5-6]}\end{array}$ & $\begin{array}{c}1 \\
{[1-} \\
1]\end{array}$ & $\begin{array}{c}1 \\
{[0.87-} \\
1.13]\end{array}$ & $\begin{array}{c}2 \\
{[1-2]}\end{array}$ & $\begin{array}{c}23 \\
{[23-} \\
23]\end{array}$ & $\begin{array}{c}24 \\
{[23.87-} \\
24.13]\end{array}$ & $\begin{array}{c}26 \\
{[25.46-26]}\end{array}$ \\
\hline $2020-04-29$ & $\begin{array}{c}9 \\
{[8-10]}\end{array}$ & $\begin{array}{c}13 \\
{[12.66-} \\
13.34]\end{array}$ & $\begin{array}{c}17 \\
{[16-} \\
18.11]\end{array}$ & $\begin{array}{c}1 \\
{[1-1]}\end{array}$ & $\begin{array}{c}3 \\
{[2.85-3.15]}\end{array}$ & $\begin{array}{c}5 \\
{[5-5]}\end{array}$ & $\begin{array}{c}1 \\
{[1-} \\
1]\end{array}$ & $\begin{array}{c}1 \\
{[0.85-} \\
1.15]\end{array}$ & $\begin{array}{c}2 \\
{[1-2]}\end{array}$ & $\begin{array}{c}22 \\
{[22-} \\
22]\end{array}$ & $\begin{array}{c}23 \\
{[22.85-} \\
23.15]\end{array}$ & $\begin{array}{c}25 \\
{[25-26]}\end{array}$ \\
\hline
\end{tabular}

Legend: Actual numbers of patients in the hospital at the specific wards and the predicted numbers for the next ten days - April 20,2020 . The predictions are made for the three different scenarios (Best Q05, Median. Worst Q90) with the Monte Carlo simulation error

hospitals have one of the larger software vendors to capture this administrative data). We presume that this step will take most of the time to set up the model for the planning tool. The need for an accurate planning tool is high, as a lack of equipment to apply oxygen related therapies, such as invasive mechanical ventilation, increases mortality [21], and so does an overflow on ICU beds [22]. An optimal organization within the hospitals is therefore needed, and we believe that prediction strategies as discussed in this paper can be helpful to obtain this objective.

The prediction accuracy of our model can in principle be further improved by making use of patient characteristics, such as age and gender, when modelling transitions between wards. We have chosen not to do this in view of the additional cost of data manipulation, the risk of model misspecification when Cox proportional hazards models are used for the cause-specific transition hazards, and the fact that such patient characteristics are unknown for future patients.

\section{Conclusions}

The proposed algorithm can be quickly setup and is an added value during the COVID-19 pandemic to predict the needed capacity within the hospital by ward type. 
Table 4 For each ward the prediction as on April 20, 2020 and April 27, 2020, for the next ten days, as reported via a best-meanworst case scenario

\begin{tabular}{|c|c|c|c|c|c|c|c|c|c|c|c|c|}
\hline \multirow[t]{3}{*}{ day } & \multicolumn{3}{|l|}{ Cohort } & \multicolumn{3}{|c|}{ ICU Midcare } & \multicolumn{3}{|c|}{ ICU Standard } & \multicolumn{3}{|c|}{ ICU Ventilated } \\
\hline & \multicolumn{2}{|l|}{ Predictions } & \multirow[t]{2}{*}{ Actual } & \multicolumn{2}{|l|}{ Predictions } & \multirow[t]{2}{*}{ Actual } & \multicolumn{2}{|c|}{ Predictions } & \multirow[t]{2}{*}{ Actual } & \multicolumn{2}{|l|}{ Predictions } & \multirow[t]{2}{*}{ Actual } \\
\hline & 20-apr & 27-apr & & 20-apr & 27-apr & & 20-apr & 27-apr & & 20-apr & 27-apr & \\
\hline April 15, 2020 & & & 36 & & & 8 & & & 0 & & & 26 \\
\hline April 16, 2020 & & & 35 & & & 8 & & & 1 & & & 25 \\
\hline April 17, 2020 & & & 41 & & & 6 & & & 0 & & & 22 \\
\hline April 18, 2020 & & & 28 & & & 7 & & & 0 & & & 23 \\
\hline April 19, 2020 & & & 24 & & & 8 & & & 1 & & & 23 \\
\hline April 20, 2020 & $24-25-27$ & & 22 & $10-10-11$ & & 9 & $1-1-1$ & & 0 & $24-24-24$ & & 24 \\
\hline April 21, 2020 & $26-28-31$ & & 19 & $8-9-10$ & & 9 & $1-1-1$ & & 1 & $24-24-25$ & & 23 \\
\hline April 22, 2020 & $28-31-34$ & & 20 & $6-7-8$ & & 4 & $1-1-1$ & & 1 & $25-25-26$ & & 24 \\
\hline April 23, 2020 & $29-32-36$ & & 19 & $5-6-8$ & & 4 & $1-1-1$ & & 1 & $26-26-27$ & & 21 \\
\hline April 24, 2020 & $26-29-33$ & & 16 & $4-5-7$ & & 5 & $1-1-2$ & & 2 & $26-26-28$ & & 21 \\
\hline April 25, 2020 & $20-23-27$ & & 14 & $2-3-6$ & & 6 & $1-1-2$ & & 2 & $25-26-27$ & & 20 \\
\hline April 26, 2020 & $14-18-22$ & & 13 & $1-3-5$ & & 8 & $1-1-2$ & & 2 & $25-26-27$ & & 20 \\
\hline April 27, 2020 & $8-12-16$ & & 14 & $1-3-5$ & & 9 & $1-1-2$ & & 1 & $25-26-28$ & & 20 \\
\hline April 28, 2020 & $10-13-18$ & $15-16-17$ & 18 & $1-3-5$ & $11-11-12$ & 7 & $1-1-2$ & $2-2-2$ & 0 & $23-24-26$ & $20-20-20$ & 15 \\
\hline April 29, 2020 & $11-15-19$ & $16-18-20$ & 17 & $1-3-5$ & $10-10-11$ & 8 & $1-1-2$ & $2-2-2$ & 1 & $22-23-25$ & $20-20-21$ & 14 \\
\hline April 30, 2020 & & $18-20-23$ & 17 & & $10-10-11$ & 6 & & $1-1-2$ & 1 & & $20-20-21$ & 11 \\
\hline May 1, 2020 & & $20-23-26$ & 20 & & $7-8-10$ & 6 & & $1-1-2$ & 1 & & $21-21-22$ & 10 \\
\hline May 2, 2020 & & $17-20-23$ & 20 & & $9-10-12$ & 8 & & $1-1-2$ & 1 & & $18-18-20$ & 9 \\
\hline May 3, 2020 & & $19-22-26$ & 21 & & $5-6-9$ & 9 & & $1-1-2$ & 1 & & $19-20-21$ & 9 \\
\hline May 4, 2020 & & $15-19-22$ & 17 & & $3-4-7$ & 8 & & $1-1-2$ & 1 & & $18-19-21$ & 9 \\
\hline May 5, 2020 & & $7-10-14$ & 16 & & $2-4-6$ & 9 & & $1-1-2$ & 0 & & $23-24-26$ & 6 \\
\hline May 6, 2020 & & $6-9-13$ & 15 & & $3-5-7$ & 8 & & $1-1-2$ & 1 & & $17-18-20$ & 6 \\
\hline May 7, 2020 & & $6-10-14$ & 16 & & $4-6-8$ & 9 & & $1-1-2$ & 1 & & $17-18-21$ & 5 \\
\hline
\end{tabular}

Legend: In the 'Actual' column we show the number of actual used beds in the hospital (at noon)

Table 5 One-day-ahead predictions - mean case scenario

\begin{tabular}{|c|c|c|c|c|c|c|c|c|c|c|c|c|}
\hline \multirow[b]{2}{*}{ actual day } & \multicolumn{3}{|l|}{ Cohort } & \multicolumn{3}{|c|}{ ICU Midcare } & \multicolumn{3}{|c|}{ ICU Standard } & \multicolumn{3}{|c|}{ ICU Ventilated } \\
\hline & Actual & prediction & diff & actual & prediction & diff & actual & prediction & diff & actual & prediction & Diff \\
\hline April 20, 2020 & 22 & & & 9 & & & 0 & & & 25 & & \\
\hline April 21, 2020 & 19 & 23 & 4 & 9 & 9 & 0 & 1 & 0 & -1 & 24 & 25 & 1 \\
\hline April 22, 2020 & 20 & 21 & 1 & 4 & 9 & 5 & 1 & 0 & -1 & 25 & 24 & -1 \\
\hline April 23, 2020 & 19 & 21 & 2 & 4 & 3 & -1 & 1 & 1 & 0 & 22 & 25 & 3 \\
\hline April 24, 2020 & 16 & 20 & 4 & 5 & 4 & -1 & 2 & 1 & -1 & 22 & 21 & -1 \\
\hline April 25, 2020 & 14 & 19 & 5 & 6 & 4 & -2 & 2 & 2 & 0 & 21 & 21 & 0 \\
\hline April 26, 2020 & 13 & 16 & 3 & 9 & 6 & -3 & 2 & 2 & 0 & 20 & 20 & 0 \\
\hline April 27, 2020 & 14 & 14 & 0 & 10 & 8 & -2 & 1 & 2 & 1 & 20 & 20 & 0 \\
\hline April 28, 2020 & 17 & 15 & -2 & 8 & 10 & 2 & 0 & 1 & 1 & 15 & 20 & 5 \\
\hline
\end{tabular}

Legend: 'Actual' refers to the actual numbers of patients on the actual day. 'Prediction' refers to the prediction number of patients for this Ward type made on the previous day. 'Diff' shows the difference between the predicted and the actual number of patients 


\section{Abbreviations}

ICU: Intensive Care Unit; PCR: Polymerase chain reaction

\section{Supplementary Information}

The online version contains supplementary material available at https://doi. org/10.1186/s12913-021-06492-3.

\section{Additional file 1.}

\section{Acknowledgements}

Not applicable.

\section{Authors' contributions}

Contributed to conception and design: SV, MD. Contributed to acquisition of data: MD, SM. Contributed to analyses of data: MD, SV. Contributed to interpretation of data: MD, SV, KE, SC, DB. Drafting the work: MD, SV, SM. Revising the paper for important intellectual content: KE, SC, DB. Final approval of the version submitted: all authors. Agreement to be accountable for all aspects of the work in ensuring that questions related to the accuracy or integrity of any part of the work are appropriately investigated and resolved: all authors. All authors have read and approved the manuscript.

\section{Funding}

No Funding.

\section{Availability of data and materials}

The data (csv file) that support the findings of this study are available from https://github.com/descheppermieke/Prediction-of-hospital-bed-capacityduring-the-COVID-19-pandemic.

\section{Declarations}

\section{Ethics approval and consent to participate}

The study was approved by the ethics committee at Ghent University Hospital (Belgian registration no. B6702020000164)

A separate informed consent was not obtained from all participants: The required data only includes the admission and discharge date per type of nursing unit (Cohort, Midcare, ICU), the length of stay, death and whether the patient has been ventilated. The data is linked in advance by an interna employee according to the principles of a Trust Party, whereby all possible identifiable data is removed or anonymised before it is delivered to the researcher. The analyzes are performed retrospectively on day -1 .

\section{Competing interests}

The authors declare no potential conflicts of interest with respect to the research, authorship, and/or publication of this article. All authors have confirmed their approval for submission of the manuscript. We confirm that the content of the manuscript has not been published, or submitted for publication elsewhere

\section{Author details}

${ }^{1}$ Strategic Policy Cell at Ghent University Hospital, C. Heymanslaan 10, 9000 Ghent, Belgium. ²Department of Public Health and Primary Care, Ghent University, C. Heymanslaan 10,9000 Ghent, Belgium. ${ }^{3}$ Department of Intensive Care Medicine, Ghent University Hospital, C. Heymanslaan 10, 9000 Ghent, Belgium. ${ }^{4}$ Department of General Internal Medicine, Ghent University Hospital, C. Heymanslaan 10, 9000 Ghent, Belgium. ${ }^{5}$ Department of Applied Mathematics, Computer Science and Statistics, Ghent University, Krijgslaan 281 S9, 9000 Ghent, Belgium. 'Department of Medical Statistics, London School of Hygiene and Tropical Medicine, Keppel Street, London WC1E 7HT, UK.

Received: 18 August 2020 Accepted: 6 May 2021

Published online: 18 May 2021

\section{References}

1. Remuzzi A, Remuzzi G. COVID-19 and Italy: what next? Lancet. 2020; 395(10231):1225-8. https://doi.org/10.1016/S0140-6736(20)30627-9.
2. Rhodes A, Ferdinande P, Flaatten H, Guidet B, Metnitz PG, Moreno RP. The variability of critical care bed numbers in Europe. Intensive Care Med. 2012; 38(10):1647-53. https://doi.org/10.1007/s00134-012-2627-8.

3. Leung K, Wu JT, Liu D, Leung GM. First-wave COVID-19 transmissibility and severity in China outside Hubei after control measures, and second-wave scenario planning: a modelling impact assessment. Lancet. 2020;395(10233): 1382-93. https://doi.org/10.1016/S0140-6736(20)30746-7.

4. Van de Voorde C, Lefèvre M, Mistiaen P, Detollenaere J, Kohn L, Van den Heede K. Assessing the management of hospital surge capacity in the first wave of the COVID-19 pandemic in Belgium. In: KCE reports. Brussels: Belgian Health Care Knowledge Centre (KCE); 2020.

5. Kuntz L, Scholtes $S$, Vera A. Incorporating efficiency in hospital-capacity planning in Germany. Eur J Health Econ. 2007;8(3):213-23. https://doi.org/1 0.1007/s10198-006-0021-6

6. Yi P, George SK, Paul JA, Lin L. Hospital capacity planning for disaster emergency management. Socio Econ Plan Sci. 2010;44(3):151-60. https:// doi.org/10.1016/j.seps.2009.11.002.

7. Litvak N, van Rijsbergen M, Boucherie RJ, van Houdenhoven M. Managing the overflow of intensive care patients. Eur J Oper Res. 2008;185(3):9981010. https://doi.org/10.1016/j.ejor.2006.08.021.

8. Ettelt S, Nolte E, Thomson S, Mays N. Capacity planning in health care: reviewing the international experience. Euro Observer. 2008;9(1):1-5.

9. Lai J, Ma S, Wang Y, Cai Z, Hu J, Wei N, et al. Factors associated with mental health outcomes among health care workers exposed to coronavirus disease 2019; 2020. p. 2574-3805. (Electronic))

10. Liang T. Handbook of COVID-19 prevention and treatment; 2020.

11. Rothstein MA. Flattening the curve, then what? In: Hastings bioethics forum; 2020

12. Jansson M, Liao X, Rello J. Strengthening ICU health security for a coronavirus epidemic. Intens Crit Care Nurs. 2020;57:102812. https://doi. org/10.1016/j.iccn.2020.102812.

13. Abrams S, Wambua J, Santermans E, Willem L, Kuylen E, Coletti P, et al. Modeling the early phase of the Belgian COVID-19 epidemic using a stochastic compartmental model and studying its implied future trajectories. medRxiv. 2021:35:100449. https://doi.org/10.1016/j.epidem.2 021.100449

14. Weissman GE, Crane-Droesch A, Chivers C, Luong T, Hanish A, Levy MZ et al. Locally informed simulation to predict hospital capacity needs during the COVID-19 pandemic. Ann Intern Med. 2020;173(1):21.

15. Stern RH. Locally informed simulation to predict hospital capacity needs during the COVID-19 pandemic. Ann Intern Med. 2020;173(8):679-80. https://doi.org/10.7326/L20-1061.

16. Fiocco M, Putter $\mathrm{H}$, van Houwelingen HC. Reduced-rank proportional hazards regression and simulation-based prediction for multi-state models. Stat Med. 2008;27(21):4340-58. https://doi.org/10.1002/sim.3305.

17. Ferguson N, Datta S, Brock G. msSurv: an R package for nonparametric estimation of multistate models. J Stat Softw. 2012;50(14):24. https://doi. org/10.1863/jss.v050.i14

18. de Wreede LC, Fiocco M. Putter H: mstate: an R package for the analysis of competing risks and multi-state models. J Stat Softw. 2011;38(7):1-30.

19. Wood S. Generalized additive models. 2nd ed. New York: Chapman and Hall/CRC; 2017. https://doi.org/10.1201/9781315370279.

20. Nyblom J. Note on interpolated order-STATISTICS. Stat Probability Lett. 1992 14(2):129-31. https://doi.org/10.1016/0167-7152(92)90076-H.

21. Vincent JL, Taccone FS. Understanding pathways to death in patients with COVID-19. Lancet Respir Med. 2020:2213-619 (Electronic)).

22. Taccone FS, Vangoethem N, Depauw R, Wittebole X, Blot K, Vanoyen H, et al. The role of organizational characteristics on the outcome of COVID-19 patients admitted to the ICU in Belgium. Lancet Regional Health - Europe. 2021;2:100019. https://doi.org/10.1016/j.lanepe.2020.100019.

\section{Publisher's Note}

Springer Nature remains neutral with regard to jurisdictional claims in published maps and institutional affiliations. 